\title{
ON SINGULARITIES OF SOLUTIONS OF CERTAIN DIFFERENTIAL EQUATIONS IN THREE VARIABLES $\left({ }^{1}\right)$
}

\author{
BY \\ STEFAN BERGMAN
}

1. Introduction. Many relations occurring in the theory of harmonic functions in two variables can be generalized to the case of other partial differential equations in the following manner.

The totality of (real) solutions $\Psi(X)$ of a linear partial differential equation forms a linear space $\mathcal{L}$. (Here we assume that $X$ varies in a fixed domain, say $\mathfrak{B})$. We can also introduce a complete set $\Psi_{\nu}(X), \nu=1,2, \cdots$ of particular solutions so that in the neighborhood $\mathfrak{N}$ of a point, say of the origin, every function $\Psi \in \mathscr{L}$ can be represented in the form

$$
\Psi(X)=\sum_{\nu=1}^{\infty} A_{\nu} \Psi_{\nu}(X), \quad X \in \mathfrak{R} .
$$

Further, by linear transformations of $\Psi_{\nu}(\boldsymbol{X})$ new systems

$$
\Phi_{\nu}(X)=\sum_{\mu=1}^{\infty} a_{\nu \mu} \Psi_{\mu}(X), \quad \nu=1,2, \cdots
$$

can be obtained possessing the property that every function $\Psi \in \mathscr{L}$ can be represented in $B$ in the form $\left({ }^{2}\right)$

$$
\Psi(X)=\sum_{\nu=1}^{\infty} B_{\nu} \Phi_{\nu}(X), \quad X \in \mathfrak{B} .
$$

(We note, however, that the system $\left\{\Phi_{\nu}(X)\right\}$ depends on the domain $\mathfrak{B}$ while $\left\{\Psi_{\nu}(X)\right\}$ is independent of $\mathfrak{B}$.) In many instances $\Psi_{\nu}(X), \nu=1,2, \cdots$ can be introduced in such a way that every individual $\Psi_{\nu}(X)$ is defined in a domain $\Im$ which includes $\mathbb{B}$ as a subdomain. In this connection the question arises as to what relations exist between the properties of the sequence $\left\{A_{\nu}\right\}$ and the properties of $\Psi(X)$, e.g., (1) how $\Psi(X)$ behaves on the boundary of $\mathfrak{B},(2)$ how far $\Psi(X)$ can be continued into $\Im$ outside of $\mathfrak{B}$ and what singularities it possesses in $\Im$, etc.

In the case of harmonic equations in two variables, many questions of

Presented to the Society, August 24, 1956; received by the editors May 25, 1956.

(1) This work was supported in part by an Office of Naval Research contract at Stanford University.

(2) E.g., if we assume that $\left\{\boldsymbol{\Phi}_{\nu}(\boldsymbol{X})\right\}$ is an orthonormal system. See $[7 ; 8]$. (Numbers in brackets refer to the bibliography.) 
this kind can be answered satisfactorily due to the fact that harmonic functions $\Psi(X), X=(x, y)$ can be mapped onto analytic functions $f(z), z=x+i y$, of a complex variable $z$. The class $\{f(z)\}$ of functions represent not only a linear space but even an algebra (i.e. not only the sum but also the product of two analytic functions is an analytic function).

If is of interest to generalize these results to the case of other linear partial differential equations. In the case of linear differential equations

$$
\frac{\partial^{2} \Psi}{\partial x^{2}}+\frac{\partial^{2} \Psi}{\partial y^{2}}+a \frac{\partial \Psi}{\partial x}+b \frac{\partial \Psi}{\partial y}+c \Psi=0,
$$

where $a, b, c$ are entire functions (when continued to the complex values of $x$ and $y$ ), one can introduce conveniently chosen subsequences $\left\{\Psi_{\nu}(X)\right\}$, of particular solutions, and show that many of the relations mentioned before are independent of the coefficients $a, b, c$ of the equation. Many of these results are based essentially on the fact that there exist operators $\mathbf{P}$ transforming analytic functions of a complex variable into (complex) solutions $\psi$ of (3). See $[2 ; 3 ; 4 ; 11 ; 12 ; 13 ; 14 ; 16]$. If we then combine "Re" (take the real part) with a conveniently chosen operator $\mathrm{P}$ generating complex solutions $\psi$ of (3), we obtain new operators transforming analytic functions $f$ of a complex variable into (real) solutions of (3). It should be stressed that while in the case of harmonic functions $\mathbf{P}$ is the identity operator, i.e., the operator which leaves the analytic function unchanged, in the case of more general differential equations it is useful for different purposes to consider for the same equation different operators $\mathrm{P}$ and to use different classes $\{\psi\}$ of complex solutions of (3).

In these investigations in the case of the differential equation (3) one can go a step forward. If we introduce $\left(^{3}\right) Z=x+i y, Z^{*}=x-i y$, as variables, then we can choose the system $\left\{\psi_{\nu}\left(Z, Z^{*}\right)\right\}\left(X=\left(Z, Z^{*}\right)\right)$ so that $\psi_{\nu}(Z, 0)=Z^{\nu}$, i.e., that in this case the sequence of coefficients $A_{\nu}$ (see (1)) coincides with the coefficients $b_{\nu 0}$ of the development

$$
\psi\left(Z, Z^{*}\right)=\sum_{\nu=0}^{\infty} \sum_{\mu=0}^{\infty} b_{\nu \mu} Z^{\nu} Z^{* \mu}, \quad b_{\nu \mu}=b_{\mu \nu},
$$

of the functions element of $\psi$ around the origin, i.e.,

$$
A_{\nu}=b_{\nu 0}, \quad \nu=0,1,2, \cdots .
$$

One of the problems arising when one attempts to continue these investigations is the question of generalization of this approach to the case of linear partial differential equations in three (real) variables. The first step in this direction is to define and study mappings, transforming solutions of

(3) We note that if $x$ and $y$ are real $Z^{*}=\bar{Z}$ (the conjugate of $Z$ ). If $x$ and $y$ are complex, $Z$ and $Z^{*}$ are two independent variables. 
linear partial differential equations into algebras of functions of one or two variables. In this case the mappings which in a number of instances have been studied have a somewhat different structure, and in order to arrive at a general theory it is useful to consider at first a number of special cases.

So far, the following linear spaces have been investigated in this connection:

(1) harmonic functions and harmonic vectors in three variables,

(2) solutions of differential equations of the form

$$
\Delta_{3} \psi+F\left(r^{2}\right) \psi=0, \quad r^{2}=x^{2}+y^{2}+z^{2}, \quad \Delta_{3} \equiv \frac{\partial^{2}}{\partial x^{2}}+\frac{\partial^{2}}{\partial y^{2}}+\frac{\partial^{2}}{\partial z^{2}}
$$

where $F\left(r^{2}\right)$ is an entire function.

In the present paper, we introduce operators which transform functions of one complex and one real variable into a class of solutions of the differential equation

$$
\frac{\partial \psi}{\partial x}+\frac{\partial^{2} \psi}{\partial y^{2}}+\frac{\partial^{2} \psi}{\partial z^{2}}+F(y, z) \psi=0
$$

where $F(y, z)$ is an entire function of two variables $\left(^{4}\right)$ (when continued to complex values of $y$ and $z$ ).

In $\S 2$ of the present paper we define an integral operator transforming functions $g(X, Z)$ of one real variable $(X)$ and one complex variable $(Z)$ into a class of (complex) solutions $\psi$ of (6) which we denote as solutions $\psi \in \mathscr{K}_{1}$ (i.e., as solutions of (6) belonging to the class $\aleph_{1}$ ).

Definition. A solution $\psi$ of the differential equation (6), whose series development at the origin $\left.{ }^{5}\right)$

$$
\begin{aligned}
\psi\left(X, Z, Z^{*}\right)= & \sum_{n=0}^{\infty} \sum_{m=0}^{\infty} \sum_{r=0}^{\infty} A_{n m r} X^{n} Z^{m} Z^{* r}, \\
& X=x, \\
Z= & \frac{1}{2}(z+i y), \\
Z^{*}= & -\frac{1}{2}(z-i y),
\end{aligned}
$$

possesses the property that

$$
A_{n 0 r}=0
$$

for $n \geqq 0, r \geqq 1$,

(4) In the paper Operators generating solutions of certain partial differential equations in three variables and their properties (to appear in the Journal of Mathematics and Mechanics) solutions of equation $\Delta_{3} \psi+F(y, z) \psi=0$ are considered.

(5) Unless the opposite is stressed, $Z$ and $Z^{*}$ are two independent (complex) variables. If we set $Z^{*}=-\bar{Z}, y$ and $z$ become real variables. 
is said to belong to the class $\left({ }^{6}\right) \varkappa_{1}$.

Using the method of infinite approximation in $\$ 2$, a representation of solutions $\psi \in \Re_{1}$ in terms of an arbitrary function $g(X, Z)$ of the real variable $(X)$ and the imaginary variable $(Z)$ is given. Applying then certain formal transformations in $\$ 2$, we obtain an integral operator transforming functions $g(X, Z)$ into solutions $\psi \in \mathcal{K}_{1}$ of the equation (6). Using this integral operator we introduce in $\S 3$ solutions $\psi \in \Re_{1}$ which possess on a given manifold $\mathfrak{R}$ singularities which we denote as pole-like singularities of the class $\mathscr{L}$. In $\S 3$ we establish some properties of these singularities.

In $\$ 4$ we give the necessary and sufficient conditions in terms of the properties of the subsequence $\left\{A_{m n 0}\right\}$ of the coefficients of the series development (7) in order that the solution $\psi=\sum A_{m n r} X^{n} Z^{m} Z^{*_{r}} \in \mathcal{K}_{1}$ of (6) has along a given curve $\left({ }^{7}\right) \mathbb{R}$ "pole-like singularities of class $\mathcal{L}$." These relations are independent of the coefficient $F(y, z)$ of the equation (6). These theorems about solutions $\psi \in K_{1}$, can be easily interpreted as corresponding results for real solutions $\Psi$ of the equation (6).

In the present paper we derive certain results referring to the connection between the coefficients or the development of a function element of a solution $\psi$ and the properties of $\psi$ in the large. It is clear that one can use an analogous procedure to derive various other results of a similar kind (compare e.g. $[4$, p. $299 \mathrm{ff}$. ]) but they are not discussed in the present paper.

The author wishes to thank Dr. M. Maschler and Dr. Johannes C. C. Nitsche for helpful criticism and various suggestions in connection with the present paper.

2. An operator transforming functions of one real and one complex variable into solutions of (2.1). Let us consider the equation $\left({ }^{8}\right)$

(1) $\widehat{L}(\widehat{\psi})=\frac{\partial^{s} \widehat{\psi}}{\partial x^{s}}+\frac{\partial^{2} \widehat{\psi}}{\partial y^{2}}+\frac{\partial^{2} \widehat{\psi}}{\partial z^{2}}+\widehat{F}(y, z) \widehat{\psi}=0, \quad \widehat{\psi} \equiv \widehat{\psi}(x, y, z), s=1,2$.

With the notation $X=x, Z=(z+i y) / 2, Z^{*}=-(z-i y) / 2, \quad F\left(Z, Z^{*}\right)$ $=\hat{F}(y, z), \psi_{s}\left(X, Z, Z^{*}\right) \equiv \widehat{\psi}(x, y, z)$ the equation (1) assumes the form

$$
L\left(\psi_{s}\right)=\frac{\partial^{s} \psi_{s}}{\partial X^{s}}-\frac{\partial^{2} \psi_{s}}{\partial Z \partial Z^{*}}+F \psi_{s}=0
$$

In this section we shall discuss properties of solutions of (2) under the assump-

(6) If we shift the origin or rotate the coordinate system the property belonging to the class $\mathscr{K}_{1}$ is not preserved.

$\left({ }^{7}\right)$ The solution $\psi$ is multivalued in the neighborhood of the singularity line $\&$ and we have to introduce branches $Z=Z \nu(X), \nu=0,1,2, \cdots, M-1$, along $\mathfrak{\&}$ in order to make the solution $\psi$ single-valued.

(8) In this section we consider the equation (1) rather than (1.1) since the results of the present section hold in the general case, $s=1$ or 2 . 
tion that $F$ is a function of $Z$ and $Z^{*}$ alone, and continuous in its arguments in some domain.

In the present section we derive for the solution $\psi_{s} \in \mathcal{K}_{1}$ certain relations which we shall use in $\S \S 3$ and 4 , namely, we shall determine the complex solution $\psi_{s}\left(X, Z, Z^{*}\right)=\mathrm{P}_{s}[g(X, Z)]$ of $(2)$ which satisfies in the "characteristic" plane, $Z^{*}=0$, the conditions

$$
\psi_{s}(X, Z, 0)=g(X, Z)
$$

and in the plane $Z=0$

$$
\psi_{8}\left(X, 0, Z^{*}\right)=g(X, 0),
$$

where $g(X, Z)$ is a given function regular analytic in $X$ and $Z$. In order to write this solution we shall introduce the following notation: Let $F_{\mu}$ $=F\left(Z_{\mu}, Z_{\mu}^{*}\right)$, then we introduce the abbreviations

$$
T_{\nu}^{(s k)}\left(\epsilon_{\nu}, \epsilon_{\nu-1}, \cdots, \epsilon_{1}\right)=\int_{0}^{Z^{*}} \int_{0}^{Z} F_{\nu}^{\epsilon_{\nu}} \int_{0}^{z_{\nu}^{*}} \int_{0}^{Z_{\nu}} F_{\nu-1}^{\epsilon_{\nu-1}} \cdots \int_{0}^{Z_{2}^{*}} \int_{0}^{Z_{2}} F_{1}^{\epsilon_{1}} g_{1}^{(s k)} \delta_{\nu},
$$

$$
\begin{aligned}
& \nu>1, \\
& \nu \geqq 1,
\end{aligned}
$$$$
\delta_{\nu}=\prod_{\mu=1}^{\nu} d Z_{\mu} d Z_{\mu}^{*}
$$

where $\epsilon_{j}=0$ or $1, j=1,2, \cdots, \nu$, and such that

$$
\epsilon_{1}+\epsilon_{2}+\cdots+\epsilon_{\nu}+k=\nu .
$$

Here $\nu$ is a positive integer, $k=0,1,2, \cdots, \nu ; s=1,2$, and $\left({ }^{9}\right)$

$$
g_{1}^{(s k)}=\frac{\partial^{s k} g\left(X, Z_{1}\right)}{\partial X^{s k}} .
$$

We introduce the sums of $T_{\nu}^{(s \boldsymbol{k})}$ by writing

$$
J_{\nu}^{(s k)}=\sum T^{(s k)}\left(\epsilon_{\nu}, \epsilon_{\nu-1}, \cdots, \epsilon_{2}, \epsilon_{1}\right), \quad \epsilon_{\nu} \equiv \underset{\epsilon_{\nu}}{k} ;
$$

here the sum is taken over all combinations of the $\epsilon_{j}$ which satisfies (6). For example, for $\nu=3$,

$$
J_{3}^{(0)}=\int_{0}^{Z^{*}} \int_{0}^{Z} F_{3} \int_{0}^{Z_{3}^{*}} \int_{0}^{Z_{3}} F_{2} \int_{0}^{Z_{2}^{*}} \int_{0}^{Z_{2}} F_{1} g_{1} \delta_{3},
$$

$$
F_{\eta} \equiv F\left(Z_{\eta}, Z_{\eta}^{*}\right), \quad g_{1} \equiv g\left(X, Z_{1}\right), \quad \delta_{3} \equiv \prod_{\mu=1}^{3} d Z_{\mu} d Z_{\mu}^{*}
$$

(9) For example, if $k=0, s=1$ or 2 , then $g^{(s k)}=g^{(0)} \equiv g(X, Z)$; if $k=1, s=1, g^{(s k)}=g^{(1)}$ $\equiv \partial g(X, Z) / \partial X$, if $k=2, s=2, g^{(s k)}=g^{(4)} \equiv \partial^{4} g(X, Z) / \partial X^{4}$, etc. 


$$
\begin{aligned}
J_{3}^{(s)}= & \int_{0}^{Z^{*}} \int_{0}^{Z} F_{3} \int_{0}^{Z_{3}^{*}} \int_{0}^{Z_{3}} F_{2} \int_{0}^{Z_{2}^{*}} \int_{0}^{Z_{2}} g_{1}^{(s)} \delta_{3}, \\
& +\int_{0}^{Z^{*}} \int_{0}^{Z} F_{3} \int_{0}^{Z_{3}^{*}} \int_{0}^{Z_{3}} \int_{0}^{Z_{2}^{*}} \int_{0}^{Z^{2}} F_{1} g^{(s)} \delta_{3} \\
& +\int_{0}^{Z^{*}} \int_{0}^{Z} \int_{0}^{Z_{3}^{*}} \int_{0}^{Z_{3}} F_{2} \int_{0}^{Z_{2}^{*}} \int_{0}^{Z_{2}} F_{1} g_{1}^{(s)} \delta_{3}, \\
J_{3}^{(2 s)}= & \int_{0}^{Z^{*}} \int_{0}^{Z} F_{3} \int_{0}^{Z_{3}^{*}} \int_{0}^{Z_{3}} \int_{0}^{Z_{2}^{*}} \int_{0}^{Z_{2}} g^{(2 s)} \delta_{3} \\
& +\int_{0}^{Z^{*}} \int_{0}^{Z} \int_{0}^{Z_{3}^{*}} \int_{0}^{Z_{3}} F_{2} \int_{0}^{Z_{2}^{*}} \int_{0}^{Z_{2}} g_{1}^{(2 s)} \delta_{3} \\
& +\int_{0}^{Z^{*}} \int_{0}^{Z} \int_{0}^{Z_{3}^{*}} \int_{0}^{Z_{3}} \int_{0}^{Z_{2}^{*}} \int_{0}^{Z_{2}} F_{1} g_{1}^{(2 s)} \delta_{3}
\end{aligned}
$$

Define $J_{0}^{(0)}=g \equiv g(X, Z), J_{1}^{(0)}=\int_{0}^{Z} \int_{0}^{Z^{*}} g\left(X, Z_{1}\right) \delta_{1}, J_{1}^{(1)}=\int_{0}^{Z} \int_{0}^{Z^{*}} F\left(Z_{1}, Z_{1}^{*}\right) g^{(s)}\left(X Z_{1}\right) \delta_{1}$. With this notation we introduce the function $\psi_{s}^{(n)}\left(X, Z, Z^{*}\right)=\sum_{\nu=0}^{n} \sum_{k=0}^{\nu} J_{\nu}^{(s k)}$ and we shall show that under certain assumptions, $\lim _{n \rightarrow \infty} \psi_{s}^{(n)}\left(X, Z, Z^{*}\right)$ exists and is the required solution. More specifically,

THEOREM 2.1. Let in a domain $\left({ }^{10}\right) \mathfrak{D}$ of the $\left(X, Z, Z^{*}\right)$-space

$$
\left|F\left(Z, Z^{*}\right)\right| \leqq C<\infty, \quad\left|g^{(s k)}\right| \equiv\left|\frac{\partial^{s k} g(X, Z)}{\partial X^{s k}}\right| \leqq A k ! \varepsilon_{s k}, \quad A<\infty .
$$

Here $A$ and $C$ are positive constants, while $\varepsilon_{s k}$ are non-negative constants. Then

$$
\psi_{s}\left(X, Z, Z^{*}\right)=\lim _{n \rightarrow \infty} \psi_{s}^{(n)}\left(X, Z, Z^{*}\right) \equiv \mathrm{P}_{s}[g(X, Z)]
$$

exists for $\left(X, Z, Z^{*}\right) \in \mathfrak{D}$, provided that $\varepsilon_{\text {sk }}$ are chosen so that the series

$$
\sum_{n=0}^{\infty} \frac{A\left|Z Z^{*}\right|^{n / 2}}{C^{n / 2}} \varepsilon_{s n} B_{n}\left[2 C^{1 / 2}\left|Z Z^{*}\right|^{1 / 2}\right]
$$

converges. Here $B_{n}(\xi)=\sum_{\nu=0}^{\infty}\left[(\xi / 2)^{n+2 \nu} / \nu !(\nu+n) !\right]$ are modified Bessel functions. See $[15$, p. 29].

Proof. From (10) it follows that

$$
\left|T_{\nu}^{(s k)}\left(\epsilon_{\nu}, \epsilon_{\nu-1}, \cdots, \epsilon_{1}\right)\right| \leqq C^{\nu-k} A k !\left|\frac{Z^{\nu} Z^{*_{\nu}}}{(\nu !)^{2}}\right| \varepsilon_{s k}
$$

(see (5)). Hence, according to (8)

$$
\text { (8) }
$$

$\left({ }^{10}\right)$ We assume that $\mathfrak{D}$ has the following property: every intersection of $\mathfrak{D}$ with the space $\left[X=c_{1}, Z^{*}=c_{2}\right]$ as well as with $\left[X=c_{3}, Z=c_{4}\right]$ is a star domain. Here $c_{k}=$ constant, $k=1,2,3,4$. 
Thus, if $\psi_{s}^{(-1)} \equiv 0$,

$$
\left|J^{(s k)}\right| \leqq\left(\begin{array}{l}
\nu \\
k
\end{array}\right) C^{\nu-k} A k ! \frac{\left|Z Z^{*}\right|^{\nu}}{(\nu !)^{2}} \varepsilon_{s k} .
$$

$$
\begin{aligned}
\mid \sum_{\eta=0}^{\infty}\left(\psi_{s}^{(\eta)}\right. & \left.-\psi_{s}^{(\eta-1)}\right)\left|\leqq \sum_{\nu=0}^{\infty} \sum_{k=0}^{\nu}\right| J_{\nu}^{(s k)} \mid \\
& \leqq \sum_{\nu=0}^{\infty} \sum_{k=0}^{\nu}\left(\begin{array}{l}
\nu \\
k
\end{array}\right) C^{\nu-k} A k ! \frac{\left|Z Z^{*}\right|^{\nu}}{(\nu !)^{2}} \varepsilon_{s k} \\
& =\sum_{k=0}^{\infty} \sum_{\nu=k}^{\infty}\left(\begin{array}{l}
\nu \\
k
\end{array}\right) C^{\nu-k} A k ! \varepsilon_{s k} \frac{\left|Z Z^{*}\right|^{\nu}}{(\nu !)^{2}}=\sum_{k=0}^{\infty} A \varepsilon_{s k} \sum_{\nu=k}^{\infty} C^{\nu-k} \frac{\left|Z Z^{*}\right| \nu}{\nu !(\nu-k) !} \\
& =\sum_{k=0}^{\infty} \frac{\left|Z Z^{*}\right|^{k / 2}}{C^{k / 2}} A \varepsilon_{s k} B_{k}\left(2\left(C\left|Z Z^{*}\right|\right)^{1 / 2}\right) .
\end{aligned}
$$

Since the last series converges (see (12)) it follows that $\psi=\lim _{n \rightarrow \infty} \psi^{(n)}$ exists.

THEOREM 2.2. If $\psi_{s}=\sum_{\nu=0}^{\infty}\left(\sum_{k=0}^{v} J_{v}^{(s k)}\right)$ converges uniformly in a domain $\mathfrak{D}$, and $\mathfrak{B}$ is the projection of $\mathfrak{D}$ on the $\left(Z, Z^{*}\right)$ space, then $\psi_{s}\left(X, Z, Z^{*}\right)$ is a solution of (2) defined in $\mathfrak{D}$, which satisfies the conditions

$$
\psi_{s}(X, Z, 0)=g(x, Z), \quad \text { (4) } \psi_{s}\left(X, 0, Z^{*}\right)=g(x, 0) .
$$

Proof. We have

$$
J_{0}^{(0)}(X, Z, 0)=g(X, Z), \quad J_{0}^{(0)}\left(X, 0, Z^{*}\right)=g(X, 0)
$$

and since $\sum_{k=0}^{\nu} J_{\nu}^{(s k)}(X, Z, 0)=\sum_{k=0}^{p} J_{\nu}^{(s k)}\left(X, 0, Z^{*}\right)=0$ for $\nu \geqq 1$, (3) and (4) follow.

Next, observe that

$$
\begin{aligned}
\int_{0}^{Z^{*}} \int_{0}^{Z}\left[\left(\partial^{s} J_{\nu}^{(s k)} / \partial X^{s}\right)+F J^{(s k)}\right] d Z d Z^{*} & \\
& =\sum T_{\nu+1}^{(s(k+1))}\left(0, \underset{\epsilon_{\nu}}{k}, \cdots, \underset{\epsilon_{1}}{k}\right)+\sum T_{\nu+1}^{(s k)}\left(1, \underset{\epsilon_{\nu}}{k}, \cdots, \underset{\epsilon_{1}^{k}}{k} .\right.
\end{aligned}
$$

Here the summation $\sum$ is taken over all $\epsilon_{j}^{k}$ satisfying the relation $\sum \epsilon_{j}^{k}=\nu-k$. Hence

$$
\begin{aligned}
\int_{0}^{Z^{*}} & \int_{0}^{Z}\left[\left(\partial^{s}\left(\sum_{k=0}^{D} J_{\nu}^{(s k)}\right) / \partial X^{s}\right)+F \sum_{k=0}^{\nu} J_{\nu}^{(s k)}\right] d Z d Z^{*} \\
& =T_{\nu+1}^{(s(v+1))}(0,0, \cdots, 0)+\sum_{k=1}^{\nu} J_{\nu+1}^{(s k)}+T_{\nu+1}^{(0)}(1,1, \cdots, 1)=\sum_{k=0}^{\nu+1} J_{\nu+1}^{(s k)},
\end{aligned}
$$

because $T_{\nu+1}^{(0)}(1,1, \cdots, 1)=J_{\nu+1}^{(0)}$ and $T_{\nu+1}^{(s(\nu+1))}(0,0, \cdots, 0)=J_{\nu+1}^{(s(\nu+1))}$. Thus

$$
\begin{aligned}
\int_{0}^{Z^{*}} \int_{0}^{Z}\left[\sum_{\nu=0}^{\infty}\left(\partial^{s}\left(\sum_{k=0}^{\nu} J_{\nu}^{(s k)}\right) / \partial X^{s}\right)\right. & \left.+\sum_{\nu=0}^{\infty}\left(\sum_{k=0}^{\nu} J_{\nu}^{(s k)}\right)\right] d Z d Z^{*} \\
& =\sum_{\nu=0}^{\infty}\left(\sum_{k=0}^{v+1} J_{\nu+1}^{(s k)}\right)=\sum_{\mu=1}^{\infty} \sum_{k=0}^{\mu} J_{\mu}^{(s k)} .
\end{aligned}
$$


Hence $\psi_{s}\left(X, Z, Z^{*}\right)$ satisfies the relation

$$
\begin{aligned}
\int_{0}^{Z^{*}} \int_{0}^{Z}\left[\left(\partial^{s} \psi_{s}\left(X, Z, Z^{*}\right) / \partial X^{s}\right)+F \psi_{s}\left(X, Z, Z^{*}\right)\right] d Z d Z^{*} & \\
& =\psi_{s}\left(X, Z, Z^{*}\right)-g(X, Z)
\end{aligned}
$$

since $J_{0}^{(0)}=g(X, Z)$.

Differentiating each side with respect to $Z^{*}$ and $Z$, we obtain

$$
\left(\partial^{s} \psi_{s}\left(X, Z, Z^{*}\right) / \partial X^{s}\right)+F \psi_{s}\left(X, Z, Z^{*}\right)=\partial^{2} \psi_{s}\left(X, Z, Z^{*}\right) / \partial Z \partial Z^{*} .
$$

Consequently $\psi_{s}$ satisfies the equation (2).

In (8), (9a), (9b), etc. the expressions $J_{\nu}^{(s k)}$ are represented in a form which is inconvenient for certain purposes; in particular, when evaluating $T_{\nu}^{(s)}$ it is necessary to integrate $\nu$ times an expression which involves the associate function $g$ or its derivatives $g^{(s k)}$. This obscures the relations between various properties of $g$ and the corresponding solution of the differential equation. It is therefore of interest to transform these integrals into expressions where it is necessary to integrate over $g$ (or $g^{(s k)}, k=1,2, \cdots, \nu$ ) only once.

Under an additional hypothesis $\left({ }^{11}\right)$ about $F$ such a representation is possible.

Hypothesis A. We assume that $F\left(Z, Z^{*}\right)$ can be represented as a finite sum of expressions, each of which is a product of an entire function of $Z$ multiplied by an entire function of $Z^{*}$, i.e., we assume that

$$
F\left(Z, Z^{*}\right)=\sum_{\mathcal{H}=1}^{N} H_{\mathfrak{H}}(Z) G_{\mathfrak{H}}\left(Z^{*}\right), \quad N<\infty,
$$

where $H_{\mathfrak{H C}}$ (resp. $G_{\mathcal{H C}}$ ), $\mathfrak{F}=1,2, \cdots, N$ are entire functions of the complex variable $Z$ (resp. $Z^{*}$ ).

Under Hypothesis $A$, the relation

$$
\begin{aligned}
& \int_{0}^{Z^{*}} \int_{0}^{Z} F_{\nu}^{\epsilon_{\nu}} \int_{0}^{Z_{\nu}^{*}} \int_{0}^{Z_{\nu}} F_{\nu-1}^{\epsilon_{\nu-1}} \cdots \int_{0}^{Z_{2}^{*}} \int_{0}^{Z_{2}} F_{1}^{\epsilon_{1}} \mathrm{~g}\left(Z_{1}\right) \delta_{\nu} \\
& =\sum_{\mathcal{F}_{\nu}=1}^{N} \sum_{\mathcal{F C}_{\nu}-1=1}^{N} \cdots \sum_{\mathcal{K C}_{1}=1}^{N} \int_{0}^{Z^{*}} \int_{0}^{Z} H_{\mathcal{H C}_{1}}^{\left[\epsilon_{\nu}\right]}\left(Z_{\nu}\right) G_{\mathcal{H C}_{\nu}}^{\left[\mathcal{E}_{\nu}\right]}\left(Z_{\nu}^{*}\right) \\
& \cdots \int_{0}^{Z_{2^{*}}} \int_{0}^{Z_{2}} H_{\mathcal{F C}_{1}}^{\left[\epsilon_{1}\right]}\left(Z_{1}\right) G_{\mathcal{F C}_{1}}^{\left[\epsilon_{1}\right]}\left(Z_{1}^{*}\right) g\left(Z_{1}\right) \delta_{v} \\
& =\sum_{\mathcal{F}_{\nu}=1}^{N} \sum_{\mathcal{K}_{\nu}=1}^{N} \cdots \sum_{\mathcal{C}_{1}=1}^{N}\left[\int_{0}^{Z} H_{\mathcal{H}_{\nu}}^{\left[\mathcal{E}_{\nu}\right]}\left(Z_{\nu}\right) \cdots \int_{0}^{Z_{2}} H_{\mathcal{H}_{1}}^{\left[\epsilon_{1}\right]}\left(Z_{1}\right) \mathrm{g}\left(Z_{1}\right) \delta_{\nu}^{(1)}\right.
\end{aligned}
$$

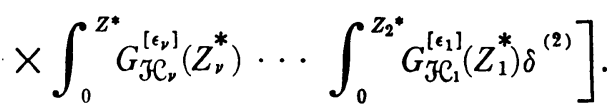

(11) In the remaining part of this section we assume that Hypothesis A holds. 
Here

$$
\begin{gathered}
H_{\mathcal{H C}_{\nu}}^{\left[\epsilon_{\nu}\right]}\left(Z_{\nu}\right)\left\{\begin{array}{l}
=H_{\mathcal{H C}_{\nu}}\left(Z_{\nu}\right), \quad G_{\mathcal{T}_{\nu}}^{\left[\epsilon_{\nu}\right]}\left(Z_{\nu}^{*}\right)=G \mathcal{K C}_{\nu}\left(Z_{\nu}^{*}\right) \text { if } \epsilon_{\nu}=1, \\
=N^{-1 / 2}, \quad \text { if } \epsilon_{\nu}=0,
\end{array}\right. \\
\delta_{\nu}^{(1)}=\prod_{\mu=1}^{\nu} d Z_{\mu}, \quad \delta_{\nu}^{(2)}=\prod_{\mu=1}^{\nu} d Z_{\mu}^{*} \text { and } \mathrm{g}(Z) \equiv g^{(s k)}(X, Z) .
\end{gathered}
$$

We consider a term

$$
L_{\nu}(Z)=\int_{0}^{Z} H_{\mathcal{K}_{\nu}}^{\left[\epsilon_{\nu}\right]}\left(Z_{\nu}\right) \cdots \int_{0}^{Z_{2}} H_{\mathcal{K}_{1}}^{\left[\epsilon_{1}\right]}\left(Z_{1}\right) \mathrm{g}\left(Z_{1}\right) \delta_{\nu}^{(1)}
$$

and we shall prove the following:

Theorem 2.3. Let $R_{\nu}(Z)=H_{\mathcal{H C}}^{\left[\epsilon_{\nu}\right]}(Z), \nu=1,2, \cdots, N$, be analytic functions of the complex variable $Z$ which are regular in a (simply connected) domain $\mathfrak{D}$. (D) includes the origin.) Then there exists an analytic function $P_{\nu}\left(Z, Z_{v}\right)$ of two complex variables $Z$ and $Z_{\nu}$ which is defined for $Z \in \mathfrak{D}, Z_{\nu} \in \mathfrak{D}$ and such that

$$
\begin{array}{rlrl}
L_{\nu}(Z) & \equiv \int_{0}^{Z} R_{\nu}\left(Z_{\nu}\right) \int_{0}^{Z_{\nu}} R_{\nu-1}\left(Z_{\nu-1}\right) \cdots \int_{0}^{Z_{2}} R_{1}\left(Z_{1}\right) \mathrm{g}\left(Z_{1}\right) \delta_{\nu}^{(1)} \\
& =\int_{0}^{Z} P_{\nu}\left(Z, Z_{\nu}\right) \mathrm{g}\left(Z_{\nu}\right) d Z_{\nu}, & Z \in \mathfrak{D}, Z_{\nu} \in \mathfrak{D},
\end{array}
$$

and the $P$, have the form

$$
P_{\nu}\left(Z, Z_{\nu}\right)=\sum_{\mu=1}^{\nu} p_{\nu}^{(\mu)}(Z) q_{\nu}^{(\mu)}\left(Z_{\nu}\right)
$$

where $p_{\nu}^{(\mu)}$ and $q_{\nu}^{(\mu)}$ are regular in $\mathfrak{D}$.

Proof. We prove our statement by induction. For $s=1$, we have by definition

$$
L_{1}(Z)=\int_{0}^{Z} R_{1}\left(Z_{1}\right) \mathrm{g}\left(Z_{1}\right) d Z_{1}
$$

so that

$$
P_{1}\left(Z, Z_{1}\right)=R_{1}\left(Z_{1}\right), \quad p_{1}^{(1)}(Z)=1, \quad q_{1}^{(1)}(Z)=R_{1}(Z) .
$$

Suppose now that our statement holds for $\nu=1,2, \cdots, n$. For the sake of brevity we shall use the symbols

$$
A_{\nu}^{(\mu)}(\eta) \equiv \int_{0}^{\eta} R_{\nu}(\zeta) p_{\nu-1}^{(\mu)}(\zeta) d \zeta .
$$


In accordance with our assumption,

$$
\begin{aligned}
L_{n+1}(Z)= & \int_{0}^{Z} R_{n+1}\left(Z_{n+1}\right) \int_{0}^{Z_{n+1}} P_{n}\left(Z_{n+1}, Z_{n}\right) \mathrm{g}\left(Z_{n}\right) d Z_{n} d Z_{n+1} \\
= & \sum_{\mu=1}^{n} \int_{0}^{Z} R_{n+1}\left(Z_{n+1}\right) p_{n}^{(\mu)}\left(Z_{n+1}\right) \int_{0}^{Z_{n+1}} q_{n}^{(\mu)}\left(Z_{n}\right) \mathrm{g}\left(Z_{n}\right) d Z_{n} d Z_{n+1} \\
= & \sum_{\mu=1}^{n} \int_{0}^{Z} \frac{\partial\left[A_{n+1}^{(\mu)}\left(Z_{n+1}\right)-A_{n+1}^{(\mu)}(Z)\right]}{\partial Z_{n+1}} \int_{0}^{Z_{n+1}} q_{n}^{(\mu)}\left(Z_{n}\right) \mathrm{g}\left(Z_{n}\right) d Z_{n} d Z_{n+1} \\
= & \left.\sum_{\mu=1}^{n}\left[A_{n+1}^{(\mu)}\left(Z_{n+1}\right)-A_{n+1}^{(\mu)}(Z)\right] \int_{0}^{Z_{n+1}} q_{n}^{(\mu)}\left(Z_{n}\right) \mathrm{g}\left(Z_{n}\right) d Z_{n}\right|_{Z_{n+1}=0} ^{Z_{n+1}-Z} \\
& -\sum_{\mu=1}^{n} \int_{0}^{Z}\left[A_{n+1}^{(\mu)}\left(Z_{n+1}\right)-A_{n+1}^{(\mu)}(Z)\right] q_{n}^{(\mu)}\left(Z_{n+1}\right) \mathrm{g}\left(Z_{n+1}\right) d Z_{n+1} \\
= & \int_{0}^{Z} P_{n+1}\left(Z, Z_{n+1}\right) \mathrm{g}\left(Z_{n+1}\right) d Z_{n+1},
\end{aligned}
$$

where

(28) $P_{n+1}\left(Z, Z_{n+1}\right)=-\left[\sum_{\mu=1}^{n} A_{n+1}^{(\mu)}\left(Z_{n+1}\right) q_{n}^{(\mu)}\left(Z_{n+1}\right)\right]+\sum_{\mu=1}^{n} A_{n+1}^{(\mu)}(Z) q_{n}^{(\mu)}\left(Z_{n+1}\right)$.

Thus $P_{n+1}\left(Z, Z_{n+1}\right)$ has the form (25) where

$$
\begin{aligned}
p_{n+1}^{(\mu)}(Z) & =A_{n+1}^{(\mu)}(Z), & q_{n+1}^{(\mu)}(Z) & =q_{n}^{(\mu)}(Z), \\
p_{n+1}^{(n+1)}(Z) & =1, & q_{n+1}^{(n+1)}(Z) & =-\sum_{\mu=1}^{n} A_{n+1}^{(n)}(Z) q_{n}^{(\mu)}(Z) .
\end{aligned}
$$

If we assume that the hypothesis A (see p. 469) holds in accordance with Theorem 2.3 each term of the form (5) can be written as

$$
\int_{0}^{Z} P^{(k)}\left(Z, Z^{*}, Z_{1}\right) g^{(s k)}\left(X, Z_{1}\right) d Z_{1}
$$

where $P^{(k)}$ is a finite sum of expressions each of which has the form

$$
\int_{0}^{Z^{*}} G_{\mathcal{H C}_{\nu}}^{\left[\epsilon_{\nu}\right]}\left(Z_{\nu}^{*}\right) \int_{0}^{Z_{\nu^{*}}} \cdots \int_{0}^{Z_{z^{*}}^{*}} G_{\mathcal{H}_{1}}^{\left[\epsilon_{1}\right]}\left(Z_{1}^{*}\right) \delta^{(2)} \times \int_{0}^{Z} P_{\nu}\left(Z, Z_{1}\right) g^{(s k)}\left(X, Z_{1}\right) d Z_{1} ;
$$

here $P_{\nu}$ is the expression introduced in Theorem 2.3 and $G_{\mathcal{J}}^{\left[\sigma_{s}\right]}$ are defined in (23). (Thus $g^{\left(k_{s}\right)}\left(X, Z_{1}\right)$ occurs in (30) in one integral only.) Therefore the

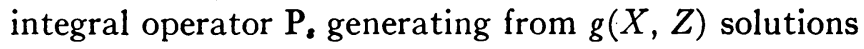

$$
\psi_{s}(X, Z)=\mathrm{P}_{\boldsymbol{s}}[g(X, Z)] \in \mathfrak{K}_{1}
$$

of equation (2) can be written in the form 


$$
\mathrm{P}_{s}(g)=\sum_{\nu=0}^{\infty}\left(\sum_{k=0}^{\nu} J_{\nu}^{(s k)}\right)=\sum_{\nu=0}^{\infty}\left(\sum_{k=0}^{\nu} \int_{0}^{Z} K^{(\nu, k)}\left(Z, Z^{*}, Z_{1}\right) g^{(s k)}\left(X, Z_{1}\right) d Z_{1}\right.
$$

where the $K^{(\nu, k)}$ are expressions which depend only on the coefficient $F$ of the equation (2) but are independent of $g(X, Z)$.

Remark 2.1. Provided that this is possible, one can rearrange the summation in (31) so that the right hand side of (31) assumes the form

$$
\sum_{k=0}^{\infty} \int_{0}^{Z} S^{(k)}\left(Z, Z^{*}, Z_{1}\right) g^{(s k)}\left(X, Z_{1}\right) d Z_{1},
$$

where the $S^{(k)}$ are independent of $g$.

3. The connection between a subsequence of the coefficients of the series development of a solution $\psi$ of (2.2) and the properties of its singularities. As mentioned in the introduction, the theory of operators permits us to use results in the theory of analytic functions of one resp. two complex variables to derive various properties of solutions of linear partial differential equations of two resp. three variables.

In this section, we shall derive relations between a subsequence of the coefficients of a series development of a solution $\bar{\psi}(x, y, z)=\psi\left(X, Z, Z^{*}\right)$ of the equation (2.1) (with $s=1$ ), and the character and location of its singularities. We note that in the case $s=1,(2.1)$ can be written also in the form

$$
\frac{\partial \psi}{\partial X}-\frac{\partial^{2} \psi}{\partial Z \partial Z^{*}}+F \psi=0
$$

The coefficient $\hat{F}(y, z)=F\left(Z, Z^{*}\right)$ of equation (1) is assumed to be an entire function of $y$ and $z$, in which case the relations mentioned above are completely independent of the special choice of the function $F$.

Before considering more general cases we note that previous results (see e.g. $[2 ; 3 ; 4])$ yield solutions of (1) possessing singularities along a given manifold:

$$
\mathfrak{R}_{1}=\left[\sum_{m=0}^{M} \sum_{n=0}^{N} a_{m n} Z^{m} Z^{*_{n}}=0, X \text { arbitrary, } a_{m n}=\bar{a}_{n m}\right],
$$

of the $x, y, z$-space. Indeed, if we write a solution $\psi$ in the form

$$
\psi\left(X, Z, Z^{*}\right)=e^{n X} \tau\left(Z, Z^{*}\right)
$$

the function $\tau\left(Z, Z^{*}\right)$ satisfies the differential equation

$$
-\tau_{Z Z^{*}}+(n+F) \tau=0
$$

in two variables and the singularities of the equations in two variables were considered in the previous papers. See $[2 ; 3 ; 4 ; 6 ; 11 ; 12 ; 16]$.

REMARK 3.1. An analogous procedure can be applied when considering the equation 


$$
\frac{\partial^{2} \psi}{\partial X^{2}}-\frac{\partial^{2} \psi}{\partial Z \partial Z^{*}}+F \psi=0,
$$

in which case instead of (4) we obtain the equation $-\tau z z^{*}+\left(n^{2}+F\right) \tau=0$, $\tau \equiv \tau\left(Z, Z^{*}\right)$.

We proceed now to the consideration of singularities along curves different from (2).

Theorem 3.1. Let $\left|F\left(Z, Z^{*}\right)\right| \leqq C<\infty$ for $|Z| \leqq C_{1},\left|Z^{*}\right| \leqq C_{1}, C$ sufficiently large, and let $\mathbb{\&}$ be the curve

$$
\mathfrak{R} \equiv\left[X-\sum_{m=0}^{M} a_{m} Z^{m}=0\right]
$$

where $X$ is a real, $Z$ is the complex variable, $Z=(z+i y) / 2$, and $a_{m}, m$ $=0,1,2, \cdots, M$ are (complex) constants, $a_{0} \neq 0$. Then

$$
\begin{gathered}
\mathrm{P}_{1}[g(X, Z)], \\
g(X, Z)=\left(X-\sum_{m=0}^{M} a_{m} Z^{m}\right)^{-\sigma}
\end{gathered}
$$

is a solution of (1) which has a singularity along the line $\mathfrak{R}$ and is regular everywhere else for $x^{2}+y^{2}+z^{2}<\infty$. Here $\sigma$ is a positive integer. (For the definition of $\mathrm{P}_{1}$ see p. 366 and p. 367).

REMARK 3.2. It follows from (5) that the curve $\mathbb{R}$ has (in general) $M$ intersection points $Z_{\nu}\left(X_{0}\right), \nu=0,1,2, \cdots, M-1$ with the plane $X=X_{0}$. (For special values of $X_{0}$ several $Z_{\nu}\left(X_{0}\right)$ may coincide.)

REMARK 3.3. In real variables $\mathfrak{R}$ is given by $\left({ }^{12}\right)$

$$
\begin{gathered}
\sum_{m=0}^{M}\left\{\frac{a_{m}^{\prime}}{2^{m}}\left[\sum_{p=0}^{[m / 2]}\left(\begin{array}{c}
m \\
2 p
\end{array}\right)(-1)^{p} z^{m-2 p} y^{2 p}\right]\right. \\
\left.-\frac{a_{m}^{\prime \prime}}{2^{m}}\left[\sum_{p=0}^{[(m-1) / 2]}(-1)^{p}\left(\begin{array}{c}
m \\
2 p+1
\end{array}\right) z^{m-1-2 p} y^{1+2 p}\right]\right\}=X, \\
\sum_{m=0}^{M}\left\{\frac{a_{m}^{\prime \prime}}{2^{m}}\left[\sum_{p=0}^{[m / 2]}(-1)^{p}\left(\begin{array}{c}
m \\
2 p
\end{array}\right) z^{m-2 p} y^{2 p}\right]\right. \\
\left.+\frac{a_{m}^{\prime}}{2^{m}}\left[\sum_{p=0}^{[(m-1) / 2]}(-1)^{p}\left(\begin{array}{c}
m \\
2 p+1
\end{array}\right) z^{m-1-2 p} y^{1+2 p}\right]\right\}=0, \\
a_{m}=a_{m}^{\prime}+i a_{m}^{\prime \prime} .
\end{gathered}
$$

(12) $[k / 2]$ denotes the largest integer which is equal or is smaller than $k / 2$. 
REMARK 3.4. Instead of the right hand side of (6a) one can use also

$$
\log \left(-X+\sum_{m=0}^{M} a_{m} Z^{m}\right)
$$

or expressions

$$
\left[\sum_{n=1}^{N} A_{n}\left(X-\sum_{m=0}^{M} a_{m} Z^{m}\right)^{n}\right]^{-1}
$$

The considerations carried out in the following hold in general also for these cases.

Proof of Theorem 3.1. I. We proceed first to the proof that our series converges at every point $X, Z$ for which $|Z| \leqq C_{1},\left|Z^{*}\right| \leqq C_{1}$ and

$$
\left|X-\sum_{m=0}^{M} a_{m} Z^{m}\right| \geqq \eta>0 .
$$

Since

$$
g^{(k)}(X, Z)=(-1)^{k} \sigma(\sigma+1) \cdots(\sigma+k-1)\left(X-\sum_{m=0}^{M} a_{m} Z^{m}\right)^{-k-\sigma}
$$

we have

$$
\left|g^{(k)}(X, Z)\right| \leqq \frac{(\sigma+k-1) !}{(\sigma-1) !} \eta^{-k-\sigma} \text { for }\left|X-\sum_{m=0}^{M} a_{m} Z^{m}\right| \geqq \eta>0 .
$$

Using (8) and repeating the consideration on p. 368 we see that

$$
\begin{aligned}
\left|\psi\left(X, Z, Z^{*}\right)\right| & \leqq \sum_{\nu=0}^{\infty} \sum_{k=0}^{\nu}\left|J_{\nu}^{(k)}\right| \\
& \leqq \sum_{\nu=0}^{\infty} \sum_{k=0}^{k=\nu} \frac{\nu !}{(\nu-k) ! k !} \frac{(k+\sigma-1) !}{(\sigma-1) !} C^{\nu-k} \eta^{-\sigma-k}\left|\frac{Z^{\nu} Z^{* \nu}}{(\nu !)^{2}}\right| \\
& =\frac{1}{(\sigma-1) !} \sum_{k=0}^{\infty} C^{-k} \frac{(., k+\sigma-1) !}{k !} \eta^{-\sigma-k} \sum_{\nu=k}^{\infty} \frac{\left|C Z Z^{*}\right|^{\nu}}{\nu !(\nu-k) !} \\
& =\frac{\eta^{-\sigma}}{(\sigma-1) !} \sum_{k=0}^{\infty} \frac{(k+\sigma-1) !}{k !}(C \eta)^{-k} \sum_{\mu=0}^{\infty} \frac{\left|C Z Z^{*}\right| \mu+k}{(\mu+k) ! \mu !} \\
& \leqq \frac{1}{(\sigma-1) ! \eta^{\sigma}} \sum_{k=0}^{\infty}\left|\frac{Z Z^{*}}{\eta}\right|^{k} \frac{(k+\sigma-1) !}{(k !)^{2}} \exp \left(\left|C Z Z^{*}\right|\right) \equiv U .
\end{aligned}
$$

For $\sigma=1$, the expression $U$ (i.e., the last line of (9)) becomes

$$
\frac{1}{\eta}\left(\exp \left|C Z Z^{*}\right|\right) \sum_{k=0}^{\infty} \frac{1}{k !}\left|\frac{Z Z^{*}}{\eta}\right|^{k}
$$


which obviously converges. From now on we shall assume that $\sigma \geqq 2$. In this case

$$
\begin{aligned}
U=\frac{1}{(\sigma-1) ! \eta^{\sigma}} & \exp \left(\left|C Z Z^{*}\right|\right)\left[\sum_{k=0}^{2 \sigma} \cdots\right. \\
& \left.+\sum_{k=2 \sigma+1}^{\infty} \frac{k !}{k !} \frac{(k+1)(k+2) \cdots(k+\sigma-1)}{k !}\left|\frac{Z Z^{*}}{\eta}\right|^{k}\right] .
\end{aligned}
$$

The first term in the bracket consists of finitely many terms; we consider the second term. We have

$$
\begin{aligned}
& \frac{(k+1)(k+2) \cdots(k+\sigma-1)}{k !} \\
& =\left(1+\frac{\sigma-1}{k-\sigma+2}\right)\left(1+\frac{\sigma-1}{k-\sigma+3}\right) \cdots\left(1+\frac{\sigma-1}{k}\right) \frac{1}{(k-\sigma+1) !} \\
& \leqq\left(1+\frac{\sigma-1}{k-\sigma+2}\right)^{\sigma-1} \frac{1}{(k-\sigma+1) !} \leqq\left(1+\frac{\sigma-1}{\sigma+3}\right)^{\sigma-1} \frac{1}{(k-\sigma+1) !} .
\end{aligned}
$$

Therefore

$$
\begin{aligned}
\sum_{k=2 \sigma+1}^{\infty} \frac{(k+1) \cdots(k+\sigma-1)}{k !}\left|\frac{Z Z^{*}}{\eta}\right|^{k} & \\
\leqq & \left(1+\frac{\sigma-1}{\sigma+3}\right)^{\sigma-1} \sum_{k=2 \sigma+1}^{\infty} \frac{1}{(k-\sigma+1) !}\left|\frac{Z Z^{*}}{\eta}\right|^{k}
\end{aligned}
$$

which obviously converges.

II. We have yet to show that the solution defined by (6) is singular at every point of $\&$, see (5).

Suppose that this were not the case, i.e., we assume that at a point $\left(X_{0}, Z_{0}, Z_{0}^{*}\right)$ of (5) the function (6) (see also (2.11)) is regular. From the relation (1) (see also (2.20)) follows that

$$
\begin{aligned}
\psi\left(X, Z, Z^{*}\right)=\left(X-\sum_{m=0}^{M} a_{m} Z^{m}\right)^{-\sigma}+\int_{0}^{Z} \int_{0}^{Z^{*}} & {\left[\left(\frac{\partial \psi\left(\xi, \zeta, \zeta^{*}\right)}{\partial \xi}\right)_{\xi=X}\right.} \\
+ & \left.F\left(\zeta, \zeta^{*}\right) \psi\left(X, \zeta, \zeta^{*}\right)\right] d \zeta d \zeta^{*}
\end{aligned}
$$

Since we assumed that $\psi$ is regular in the neighborhood of the point $\left(X_{0}, Z_{0}\right.$, $\left.Z_{0}^{*}\right)$ we can connect the point $\left(X_{0}, 0,0\right)$ with the point $\left(X_{0}, Z_{0}, Z_{0}^{*}\right)$ by a curve lying in the plane $\xi=X_{0}$ which lies in the regularity domain of $\psi\left(X_{0}, \zeta, \zeta^{*}\right)$. Consequently the second term on the right hand side of (14) will be regular at the point $\left(X_{0}, Z_{0}, Z_{0}^{*}\right)$. The term $\left(X-\sum_{m=0}^{M} a_{m} Z^{m}\right)^{-\sigma}$ is singular at this point so that the right hand side of (14) becomes singular there. But according to our assumption, the left hand side of (14) is regular there. This contra- 
diction shows that our assumption that $\psi$ is regular at $\left(X_{0}, Z_{0}, Z_{0}^{*}\right)$ was wrong.

We proceed now to a more detailed consideration of the behavior of our function in the neighborhood of a singularity $\mathfrak{R}$. In particular, we are interested in showing that for a certain approach to $\mathfrak{l}$, our function or its derivative becomes infinite. We consider now the case where

$$
\psi(X, Z, 0)=-\log \left(-X+\sum_{m=0}^{M} a_{m} Z^{m}\right) .
$$

In this case $P_{1}(g)$ (see pp. 266-268) can be written in the form

$$
\psi\left(X, Z, Z^{*}\right)=\mathrm{P}_{1}(g) \equiv J_{1}\left(X, Z, Z^{*}\right)+J_{2}\left(X, Z, Z^{*}\right)
$$

where $\left({ }^{13}\right)$

$$
\begin{aligned}
J_{1}= & -\log \left(-X+\sum a_{m} Z^{m}\right)+\int_{0}^{Z} \int_{0}^{Z^{*}} \frac{\delta_{1}}{\left(-X+\sum a_{m} Z^{m}\right)} \\
& +\sum_{n=2}^{\infty} \int_{0}^{Z} \int_{0}^{Z^{*}} \int_{0}^{Z_{n}} \int_{0}^{Z_{n}^{*}} \cdots \int_{0}^{Z_{2}} \int_{0}^{Z_{2}^{*}} \frac{(n-1) ! \delta_{n}}{\left(-X+\sum a_{m} Z_{1}^{m}\right)^{n}}, \\
\sum \equiv & \sum_{m=0}^{M},
\end{aligned}
$$

see (2.5), and

(17b)

$$
\begin{aligned}
J_{2}= & -\int_{0}^{Z} \int_{0}^{Z^{*}} F_{1} \log \left(-X+\sum a_{m} Z_{1}^{m}\right) \delta_{1} \\
& +\int_{0}^{Z} \int_{0}^{Z^{*}} F_{2} \int_{0}^{z_{2}^{*}} \int_{0}^{Z_{2}^{*}} \frac{\delta_{2}}{\left(-X+\sum a_{m} Z_{1}^{m}\right)} \\
& +\int_{0}^{Z} \int_{0}^{Z^{*}} \int_{0}^{Z^{*}} \int_{0}^{z_{2}^{*}} \frac{F_{1} \delta_{2}}{\left(-X+\sum a_{m} Z_{1}^{m}\right)} \\
& -\int_{0}^{Z} \int_{0}^{z^{*}} F_{2} \int_{0}^{Z_{2}} \int_{0}^{Z_{2}^{*}} F_{1} \log \left(-X+\sum a_{m} Z_{1}^{m}\right) \delta_{2}+\cdots,
\end{aligned}
$$

see $(2.9 a)-(2.9 c)$.

Before considering the general case it is of interest to consider a special case, namely where

$$
\sum a_{m} Z^{m}=a_{0}-Z
$$

is a linear function of $Z$, and we shall investigate the term $J_{1}\left(X, Z, Z^{*}\right)$. If we write

(13) $J_{1}$ are terms of the series which are independent of $F$ while $J_{2}$ are all remaining terms. 


$$
Y=a_{0}-X
$$

we have for $\left|Z_{\nu}\right|<|Y|$

$$
\left(Y-Z_{\nu}\right)^{-\nu-1}=Y^{-\nu-1}\left[1+\left(\begin{array}{c}
\nu+1 \\
1
\end{array}\right)\left(\frac{Z_{\nu}}{Y}\right)+\left(\begin{array}{c}
\nu+2 \\
2
\end{array}\right)\left(\frac{Z_{\nu}}{Y}\right)^{2}+\cdots\right]
$$

Thus for $\left|Z_{\nu}\right| \leqq \alpha<|Y|$ one obtains for the integrand of $J_{1}$ a uniformly convergent series. We can continue the integrand to the complex values of $y$ and $z$ (i.e. assume that $Z_{\nu}$ and $Z_{\nu}^{*}$ are two independent variables). If for a fixed $Z_{\mu}^{*}$ we carry out the integration with respect to $Z_{\mu}$ along a straight line $Z_{\mu} \equiv\left|Z_{\mu}\right| e^{i c_{1}}, c_{1}$ constant, we have

$$
\left|d Z_{\mu}\right|=d Z_{\mu}, \quad Z_{\mu}=\left|Z_{\mu}\right| .
$$

Similarly, for $Z_{\mu}^{*}=\left|Z_{\mu}^{*}\right| e^{i c_{2}}, c_{2}$ constant,

$$
\left|d Z_{\mu}^{*}\right|=d Z_{\mu}^{*}, \quad Z_{\mu}^{*}=\left|Z_{\mu}^{*}\right|
$$

If we develop $J_{1}$ for $\left|Z_{\mu}\right|<|Y|$ into a power series and integrate term by term, we obtain

$$
\begin{aligned}
J_{1}= & -\log (Y-Z)-Z^{*} \log \left(1-\frac{Z}{Y}\right) \\
& +\sum_{n=2}^{\infty}\left[-\log \left(1-\frac{Z}{Y}\right)-\frac{Z}{Y}-\frac{1}{2} \frac{Z^{2}}{Y^{2}} \cdots-\frac{1}{n-1} \frac{Z^{n-1}}{Y^{n-1}}\right] \frac{Z^{* n}}{n !} \\
= & -\log Y+\sum_{n=1}^{\infty} \frac{1}{n}\left(\frac{Z}{Y}\right)^{n}+Z^{*} \sum_{n=1}^{\infty} \frac{1}{n}\left(\frac{Z}{Y}\right)^{n}+\frac{Z^{* 2}}{2 !} \sum_{n=2}^{\infty} \frac{1}{n}\left(\frac{Z}{Y}\right)^{n} \\
& +\frac{Z^{* 3}}{3 !} \sum_{n=3}^{\infty} \frac{1}{n}\left(\frac{Z}{Y}\right)^{n}+\cdots \\
= & -\log Y+\exp Z^{*} \sum_{N=1}^{\infty}\left(\frac{1}{N}\right)\left(\frac{Z}{Y}\right)^{N}-\sum_{N=1}^{\infty} \frac{1}{N}\left(\frac{Z}{Y}\right)^{N}\left(\sum_{n=N+1}^{\infty} \frac{Z^{* n}}{n !}\right) \\
\equiv & \mathrm{P}_{1}-\mathrm{P}_{2} .
\end{aligned}
$$

Therefore if we approach $Z \rightarrow Y$ and $Z^{*}=-\bar{Z} \rightarrow-\bar{Y}$ (or any other values $\left.Z^{*} \neq \infty\right)$ then

$$
\lim _{Z \rightarrow Y} J_{1}\left(X, Z, Z^{*}\right)=\infty .
$$

Indeed, for $Z \rightarrow Y$ and $Z^{*}=-\bar{Z} \rightarrow-\bar{Y}, P_{1}(X, Z, \bar{Z}) \rightarrow \infty$. For $|Z / Y| \leqq 1$ we have 


$$
\begin{aligned}
\left|\mathrm{P}_{2}\left(X, Z, Z^{*}\right)\right| & \leqq\left|\sum_{n=1}^{\infty} \frac{1}{n}\left[\frac{\left|Z^{*}\right| n+1}{(n+1) !}+\frac{\left|Z^{*}\right| n+2}{(n+2) !}+\cdots\right]\right| \\
& \leqq\left|\sum_{n=2}^{\infty}\left(\sum_{k=1}^{n-1} \frac{1}{k}\right) \frac{\left|Z^{*}\right|^{n}}{n !}\right| \leqq\left|\sum_{n=2}^{\infty} \frac{\left|Z^{*}\right|{ }^{n}}{(n-1) !}\right|<\infty .
\end{aligned}
$$

REMARK 3.5. From the proof it follows that $\mathrm{P}_{1}\left(X+a_{0}-Z\right)$ becomes infinite if we approach $\left[X=a_{0}-Z\right]$ remaining in the real space (i.e., for $X$ real, $\left.Z^{*}=-\bar{Z}\right)$.

We proceed to the derivation of an upper bound for $J_{2}$. We have (concerning the notation, compare (19), (21a), (21b)),

(24) $\operatorname{Max}\left[\left|\log \frac{1}{Y-Z}\right|,\left|\frac{(p-1) !}{(Y-Z)^{p}}\right|, p=1,2, \cdots, m-1\right] \leqq \frac{(m-2) !}{(\mathrm{Y}-\mathrm{Z})^{m-1}}$

for $\left.{ }^{14}\right) 0<|Y-Z|<\rho, \rho$ sufficiently small $(Y=|Y|, Z=|Z|)$. (We use the fact that every intersection $X=$ const.

$$
\left.\left|\log \frac{1}{Y-Z}\right| \leqq \log \left|\frac{1}{Y-Z}\right|+2 \pi<\frac{1}{|Y-Z|}, \text { for }|Y-Z|<\rho .\right)
$$

Further, if the constant $\tilde{F}$ is chosen so that

$$
\operatorname{Max}[1,|F|] \leqq \tilde{F}
$$

then

$$
\begin{aligned}
\mid \int_{0}^{Z^{*}} & \int_{0}^{Z} F_{m}^{\epsilon_{m}} \int_{0}^{Z_{m}^{*}} \int_{0}^{Z} F_{m-1}^{\epsilon_{m-1}} \cdots \int_{0}^{Z_{2}^{*}} \int_{0}^{Z_{2}} F_{1}^{\epsilon_{1}} \frac{(p-1) ! \delta_{m}}{\left(Y-Z_{1}\right)^{p}} \mid \\
& \leqq \tilde{F}^{m} \int_{0}^{\mathrm{Z}^{*}} \int_{0}^{\mathrm{Z}} \int_{0}^{\mathrm{Z}_{m}^{*}} \int_{0}^{\mathrm{Z}_{m}} \cdots \int_{0}^{\mathrm{Z}_{2}^{*}} \int_{0}^{\mathrm{Z}_{2}} \frac{(m-2) !}{\left(\mathrm{Y}-\mathrm{Z}_{1}\right)^{m-1}} \prod_{s=1}^{m} d \mathrm{Z}_{s} d \mathrm{Z}_{s}^{*} \\
& \leqq \tilde{F}^{m} \int_{0}^{\mathrm{Z}^{*}} \int_{0}^{\mathrm{Z}} \cdots \int_{0}^{\mathrm{Z}_{3}^{*}} \int_{0}^{\mathrm{Z}_{3}}\left[\frac{(m-3) !}{\left(\mathrm{Y}-\mathrm{Z}_{2}\right)^{m-2}}-\frac{(m-3) !}{\mathrm{Y}^{m-2}}\right] \mathrm{Z}_{2}^{*} \prod_{s=2}^{m} d \mathrm{Z}_{s} d \mathrm{Z}_{s}^{*} \\
(26) & \leqq \tilde{F}^{m} \int_{0}^{\mathrm{Z}^{*}} \int_{0}^{\mathrm{Z}} \cdots \int_{0}^{\mathrm{Z}_{3}^{*}} \int_{0}^{\mathrm{Z}_{3}} \frac{(m-3) !}{\left(\mathrm{Y}-\mathrm{Z}_{2}\right)^{m-2}} \mathrm{Z}_{2}^{*} \prod_{s=2}^{m} d \mathrm{Z}_{s} d \mathrm{Z}_{s}^{*} \\
& \leqq \tilde{F}^{m} \int_{0}^{\mathrm{Z}^{*}} \int_{0}^{\mathrm{Z}} \int_{0}^{\mathrm{Z}_{m}^{*}} \int_{0}^{\mathrm{Z} m} \frac{(m-2) !\left(\mathrm{Y}-\mathrm{Z}_{m-1}\right)}{\left(m \mathrm{Z}_{m} d \mathrm{Z}_{m}^{*} d \mathrm{Z}_{m-1} d \mathrm{Z}_{m-1}\right.} \\
& \leqq \tilde{F}^{m} \int_{0}^{\mathrm{Z}} \int_{0}^{\mathrm{Z}^{*}}\left[-\log \left(1-\frac{\mathrm{Z}_{m}}{\mathrm{Y}}\right)\right]_{\left.\frac{\mathrm{Z}_{m}^{*}}{(m-1}-1\right) !}^{\left(m-\mathrm{Z}_{m} d \mathrm{Z}_{m}^{*}\right.} \\
& =\tilde{F}^{m}\left[\left(\mathrm{Y}-\mathrm{Z}^{*}\right) \log \left(1-\frac{\mathrm{Z}}{\mathrm{Y}}\right)+\mathrm{Z}\right] \frac{\mathrm{Z}^{* m}}{m !} \cdot
\end{aligned}
$$

Hence there are $\left(2^{n}-1\right)$ terms of $J_{2}$, see $(17 \mathrm{~b})$ involving $2 n$ integrations, and in each $F_{k}=F\left(Z_{k}, Z_{k}^{*}\right)$ appears at most $n$ times, in accordance with (25), the

(14) This means that the inequalities hold only in a certain neighborhood of the line $\alpha^{0}-X-Z=0$. 
sum of the terms involving $2 n$ integrations of $J_{2}$ will be majorized by

$$
2^{n} \tilde{F}^{n}\left[(\mathrm{Y}-\mathrm{Z}) \log \left(1-\frac{\mathrm{Z}}{\mathrm{Y}}\right)+\mathrm{Z}\right] \frac{\mathrm{Z}^{*_{n}}}{n !} .
$$

Consequently, $J_{2}\left(X, Z, Z^{*}\right)$ will be majorized by the sum of these terms, i.e., we have

$$
\left|J_{2}\left(X, Z, Z^{*}\right)\right| \leqq\left[(\mathrm{Y}-\mathrm{Z}) \log \left(1-\frac{\mathrm{Z}}{\mathrm{Y}}\right)+\mathrm{Z}\right] \exp \left(2 \tilde{F} \mathrm{Z}^{*}\right)
$$

which is uniformly bounded for $Z \rightarrow Y$, i.e., in particular in the neighborhood of the singular line.

This completes the proof of our assertion. In this way we obtain

THEOREM 3.2. The function $\psi\left(X, Z, Z^{*}\right)=\mathrm{P}_{1}\left[-\log \left(a_{0}-X-Z\right)\right]$ has the property that if we approach the point $Z=X-a_{0},\left|Z^{*}\right| \leqq C<\infty$ so that $\left|Z /\left(a_{0}-X\right)\right|<1, \psi\left(X, Z, Z^{*}\right)$ goes to $\infty$.

We return now to the consideration of the general case, namely to the investigation of the behavior of the solution (6) in the neighborhood of the singular line $\ell=0$, see (5), in the case where $\sigma$ is an arbitrary (positive) integer.

THEOREM 3.3. Let us assume that we approach a point $T=\left(x_{0}, y_{0}, z_{0}\right)$ lying on (5) along $a$ path (OT) lying in plane $X=X_{0}=$ const. of finite length. Here $O=\left(X_{0}, 0,0\right)$. Then at least one of the functions

$$
\begin{aligned}
\psi\left(X, Z, Z^{*}\right) & =\mathrm{P}_{1}[g(X, Z)] \text { or } \frac{\partial \psi\left(X, Z, Z^{*}\right)}{\partial X},\left|Z^{*}\right| \leqq c<\infty, \\
g(X, Z) & =\left(X-\sum_{m=0}^{M} a_{m} Z^{m}\right)^{-\sigma},
\end{aligned}
$$

cannot in absolute value on (OT) remain smaller than $A D^{-(\sigma / 2)+e}$, where $\epsilon$ is a positive (arbitrarily small) constant, $A$ a constant, and

$$
D=|L|^{2}=\left\{\left[X-\operatorname{Re}\left(\sum_{m=0}^{M} a_{m} Z^{m}\right)\right]^{2}+\left[\operatorname{Im}\left(\sum_{m=0}^{M} a_{m} Z^{m}\right)\right]^{2}\right\} .
$$

Proof. Let us assume that when we approach $T$ along $(O T)$ we have always

$$
\left|\psi\left(X, Z, Z^{*}\right)\right| \leqq A D^{-(\sigma / 2)+\epsilon,}, \quad\left|\frac{\partial \psi\left(X, Z, Z^{*}\right)}{\partial X}\right| \leqq A D^{-(\sigma / 2)+\epsilon}, \quad \epsilon>0 .
$$

Then from (14) it follows that, for $X=X_{0}$,

$$
\begin{aligned}
\frac{1}{\left|X-\sum_{m=0}^{M} a_{m} Z^{m}\right|^{\sigma}} \leqq\left|\psi\left(X, Z, Z^{*}\right)\right| \\
+\left|\int_{0}^{Z} \int_{0}^{Z^{*}}\left[\frac{\partial \psi\left(X, \zeta, \zeta^{*}\right)}{\partial X}+F\left(\zeta, \zeta^{*}\right) \psi\left(X, \zeta, \zeta^{*}\right)\right] d \zeta d \zeta^{*}\right| .
\end{aligned}
$$


For every path $(O T)$ of finite length there exist point sets $T_{\nu}=\left(y_{\nu}, z_{\nu}\right)$, $Z^{(\nu)}=z_{\nu}+i y_{\nu}, \nu=1,2, \cdots$ lying on $(O T)$ and converging to $T$ such that the minimum of $D$ on each segment $\left(O T_{\nu}\right)$ is assumed at the endpoint $T_{\nu}$ (the property $B)$. Indeed, if $D$ assumes its minimum on $\left(O T_{v}\right)$ at the point $T_{v}^{\prime}$ different from the endpoint $T_{\nu}$, we replace the segment $\left(O T_{\nu}\right)$ by $\left(O T_{\nu}^{\prime}\right)$. In this way we obtain intervals $\left(O T_{\nu}^{\prime}\right), \nu=1,2, \cdots$ possessing the desired property $B$. On the other hand, to every closed interval $(O P)$ of $(O T)$ which does not include the endpoint $T$, there exists a constant $a>0$, such that $D \geqq a>0$ on $(O P)$. Since the values of $D$ at the points $T_{\nu}$ and therefore also at the points $T_{\nu}^{\prime}$, go to 0 , the points $T_{\nu}^{\prime}$ for sufficiently large $\nu$ must lie outside the interval $(O P)$. Since we can assume that $(P T)$ can be made arbitrarily small, the set $T_{\nu}^{\prime}$ must converge to $T$.

In the following, we shall assume that the originally chosen set $T_{\nu}$ has the desired property $B$. Then, for $X=X_{0}$

$$
\begin{aligned}
& \left|\psi\left(X, \zeta, \zeta^{*}\right)\right| \leqq A\left[D\left(X, \zeta, \zeta^{*}\right)\right]^{-(\sigma / 2)+e} \leqq A D_{\nu}^{-(\sigma / 2)+\epsilon} \text { for }\left(\zeta, \zeta^{*}\right) \in(O T)_{\nu} \\
& \left|\frac{\partial \psi\left(X, \zeta, \zeta^{*}\right)}{\partial X}\right| \leqq A[D(X, \zeta, \zeta)]^{-(\sigma / 2)+\epsilon} \leqq A D_{\nu}^{-(\sigma / 2)+\nu}, \\
& D_{\nu} \equiv D\left(X, Z^{(\nu)}, Z^{*(\nu)}\right) .
\end{aligned}
$$

From (14), (32), (33) and (34) follows that

$$
\frac{1}{\left|X-\sum_{m=0}^{M} a_{m} Z^{(\nu)^{m}}\right|^{\sigma}} \leqq A D_{\nu}^{-(\sigma / 2)+\epsilon}+A D_{\nu}^{-(\sigma / 2)+\epsilon}[1+\tilde{F}] \tilde{L},
$$

where $\widetilde{F}=\max \left[F\left(\zeta, \zeta^{*}\right)\right]$ on the curve $O T$ and $\widetilde{L}=\left|\int_{0}^{Z} \int_{0}^{Z^{*}} d \zeta d \zeta^{*}\right|$.

From (35) we obtain

$$
1 \leqq D_{v}^{\epsilon} A[1+(1+\tilde{F}) \tilde{L}] .
$$

When the point $\left(X_{0}, Z^{(\nu)}, Z^{*(\nu)}\right)$ approaches $T \in(\mathbb{R}=0), \quad\left(X_{0}=\right.$ constant $)$, $D \rightarrow 0$. Since $\tilde{L}$ and $\widetilde{F}$ are uniformly bounded, the right hand side of (36) goes to zero for $\left(X_{0}, Z^{(v)}, Z^{*(\nu)}\right) \rightarrow T$. But this is a contradiction since the left-hand side always equals 1 .

REMARK 3.6. The statement of Theorem 3.3 holds if we approach the point $(X, Z, \bar{Z})=(x, y, z)$ remaining in the real $x, y, z$-space, since we can repeat the proof assuming that $Z^{*}=-\bar{Z}$.

In Theorem 3.3, we obtained a lower limit for the $\max [|\psi|,|\partial \psi / \partial X|]$ when approaching the singularity curve $\mathbb{R}$.

We proceed now to the derivation of an upper limit for $|\psi|$ when we approach $\&$. For every $X$ the curve $\&$, (see (5)), has (in general) $M$ intersection points, say $Z_{\nu}(X), \nu=0,1, \cdots, M-1$, with the plane $X=$ const. In the following, in addition, we shall assume that on the segment $\left[0, Z_{0}(X)\right]$ of the 
straight line connecting the origin 0 with $Z_{0}(X)$ there are no other roots $Z_{\nu}(X), \nu=1,2, \cdots, M-1$ of $(5)$. If $Z_{0}(X)$ has the above property and $Z_{0}(X)$ is a simple root of (5), we shall say that $Z_{0}(X)$ satisfies Condition $C$.

Theorem 3.4. If the root $Z_{0}(X)$ of (5) satisfies Condition $C$, the function

$$
\begin{aligned}
\psi\left(X, Z, Z^{*}\right)= & \frac{1}{[L(X, Z)]^{\sigma}} \\
& +\left[-\int_{0}^{Z} \int_{0}^{Z^{*}} \frac{\sigma d Z_{1} d Z_{1}^{*}}{\left[L\left(X, Z_{1}\right)\right]^{\sigma+1}}+\int_{0}^{Z} \int_{0}^{Z^{*}} \frac{F_{1} d Z_{1} d Z_{1}^{*}}{\left[L\left(X, Z_{1}\right)\right]^{\sigma}}\right] \\
& +\left[\int_{0}^{Z} \int_{0}^{Z_{1}^{*}} \int_{0}^{Z_{1}} \int_{0}^{Z_{1}^{*}} \frac{\sigma(\sigma+1) d Z_{1} d Z_{1}^{*} d Z_{2} d Z_{2}^{*}}{\left[L\left(X, Z_{2}\right]^{\sigma+2}\right.}\right. \\
& -\int_{0}^{Z} \int_{0}^{Z^{*}} F_{1} \int_{0}^{Z_{1}} \int_{0}^{Z_{1}^{*}} \frac{\sigma d Z_{1} d Z_{1}^{*} d Z_{2} d Z_{2}^{*}}{\left[L\left(X, Z_{2}\right)\right]^{\sigma+1}} \\
& -\int_{0}^{Z} \int_{0}^{Z^{*}} \int_{0}^{Z_{1}} \int_{0}^{Z_{1}^{*}} \frac{F_{2} \sigma d Z_{1} d Z_{1}^{*} d Z_{2} d Z_{2}^{*}}{\left[L\left(X, Z_{2}\right)\right]^{\sigma+1}} \\
& \left.+\int_{0}^{Z} \int_{0}^{Z^{*}} F_{1} \int_{0}^{Z_{1}} \int_{0}^{Z_{1}^{*}} \frac{F_{2} d Z_{1} d Z_{1}^{*} d Z_{2} d Z_{2}^{*}}{\left[L\left(X, Z_{2}\right)\right]^{\sigma}}\right]+\cdots, \\
F_{\nu} & \equiv F\left(Z_{\nu}, Z_{\nu}^{*}\right), \quad L(X, Z)=X-\sum_{m=0}^{M} a_{m} Z^{m}
\end{aligned}
$$

(see also (17a) and (17b)) has the property that

$$
\lim _{Z \rightarrow Z_{0}(X)}\left|\left(Z-Z_{0}(X)\right)^{\sigma} \psi\left(X, Z, Z^{*}\right)\right| \leqq \mathbf{P}^{\sigma} \exp \left(2\left|Z_{0}^{*}(X)\right| \mathbf{P} \tilde{F}\right) .
$$

Here $\mathbf{P}=1 /\left|a_{M}\right| \mu^{M-1}$ where $\mu$ is the minimal distance between the remaing zeros $Z_{\nu}(X), \nu=1,2, \cdots, M-1$ of $L=0$ and the segment $\left[0, Z_{0}(X)\right]=$ connecting 0 with $Z_{0}(X)$. Further, $\widetilde{F}=\max \left[\left|F\left(Z, Z^{*}\right)\right|, 1\right],\left(Z, Z^{*}\right) \in\left[0, Z_{0}(X)\right]$.

REMARK 3.7. We assume here that $\left|Z^{*}\right|$ remains always bounded. In particular, (38) holds if we approach $Z_{0}(X)$ in the real plane (i.e. assuming that $y$ and $z$ are real).

Proof. For an $X$, satisfying the conditions of the theorem, for all $Z \in\left[0, Z_{0}(X)\right]$, the inequality

$$
\left|X-\sum a_{m} Z^{m}\right| \geqq \mathbf{P}^{-1}\left|Z-Z_{0}(X)\right|, \quad \sum \equiv \sum_{m=0}^{M},
$$

holds, where $\mathbf{P}$ is a positive conveniently chosen constant. Indeed, according to the hypotheses of the theorem on the segment $\left[0, Z_{0}(X)\right]$ there are no more zeros $Z_{\nu}(X), \nu=1,2, \cdots, M-1$ of (5). Therefore, if we denote by $\mu$ the minimum distance between the points of $\left[0, Z_{0}(X)\right]$ and the nearest root $Z_{\nu}(X), \nu=1,2, \cdots, M-1$, then 


$$
\frac{\left|Z-Z_{0}(X)\right|}{\left|X-\sum a_{m} Z^{m}\right|}=\frac{\left|Z-Z_{0}(X)\right|}{\left|a_{M}\right|\left|Z-Z_{0}(X)\right| \prod_{\nu=1}^{M-1}\left|Z-Z_{\nu}(X)\right|} \leqq \frac{1}{\left|a_{M}\right| \mu^{M-1}}
$$

and therefore we can choose

$$
\mathbf{P}=\left(\left|a_{M}\right| \mu^{M-1}\right)^{-1} .
$$

When integrating along the straight line $\left[0, Z_{0}(X)\right]$ from 0 to an interior point $Z$ of $\left[0, Z_{0}(X)\right]$, we have

$$
\left|\int_{0}^{Z} \frac{\sigma d Z}{\left(X-\sum a_{m} Z^{m}\right)^{\sigma+1}}\right| \leqq\left|\int_{0}^{Z} \frac{\sigma d Z \mathbf{P}^{\sigma+1}}{\left|Z-Z_{0}(X)\right|^{\sigma+1}}\right| \leqq \frac{\mathbf{P}^{\sigma+1}}{\left|Z-Z_{0}(X)\right|^{\sigma}} .
$$

Similarly, we obtain

(40a) $\left|\int_{0}^{Z} \cdots \int_{0}^{Z_{n-1}} \frac{\sigma(\sigma+1) \cdots(\sigma+n-1) d Z_{n} \cdots d Z_{1}}{\left(X-\sum a_{m} Z^{m}\right)^{\sigma+n}}\right| \leqq \frac{\mathbf{P}^{\sigma+n}}{\left|Z-Z_{0}(X)\right|^{\sigma}}$

and

$$
\begin{aligned}
& \mid \int_{0}^{Z^{*}} \cdots \int_{0}^{Z_{n-1}^{*}} \int_{0}^{Z} \cdots \int_{0}^{Z_{n-1}} \\
& \quad \frac{\sigma(\sigma+1) \cdots(\sigma+n-1) d Z_{n} \cdots d Z_{1} d Z_{n}^{*} \cdots d Z_{1}^{*}}{\left(X-\sum a_{m} Z^{m}\right)^{\sigma+n}} \mid \leqq \frac{\mathbf{P}^{\sigma+n} \mid Z^{*} !^{n}}{n !\left|Z-Z_{0}(X)\right|^{\sigma}} .
\end{aligned}
$$

Since in the $n$th bracket [..] of (37) there are $2^{n}$ terms involving $2 n$ integrations, the $n$th bracket $[\ldots]$ is equal or smaller than

$$
\frac{2^{n} \mathbf{P}^{\sigma+n} \tilde{F}^{n}\left|Z^{*}\right|^{n}}{n !\left|Z-Z_{0}(x)\right|^{\sigma}}
$$

Consequently

$$
\left|\psi\left(X, Z, Z^{*}\right)\right| \leqq \sum_{n=0}^{\infty} \frac{2^{n} \mathbf{P}^{\sigma+n} \tilde{F}^{n}\left|Z^{*}\right|^{n}}{n !\left|Z-Z_{0}(X)\right|^{\sigma}}=\frac{\mathbf{P}^{\sigma}}{\left|Z-Z_{0}(X)\right|^{\sigma}} \exp \left(2\left|Z^{*}\right| P \widetilde{F}\right)
$$

from which (38) follows.

4. Conditions for the coefficients of a series development of a solution $\psi \in \mathcal{K}_{1}$ of $(2.2), s=1$, in order that $\psi$ has singularities of a certain type. In the case of functions of one complex variable there exist various results about connections between the properties of the sequence $\left\{A_{n}\right\}, n=0,1,2, \cdots$ of the series development $\sum_{n=0}^{\infty} A_{n} Z^{n}$ of an analytic function $g(Z)$ and the location and properties of its singularities. These relations can be formulated also as the corresponding relations for harmonic functions. In this form they can be generalized for the equations 


$$
\Delta \psi+A \psi_{x}+B \psi_{y}+C \psi=0,
$$

where $A, B, C$ are entire functions of $x$ and $y$. Many of these relations are either completely independent of the coefficients $A, B, C$ or depend only upon some properties of $A, B, C$. See $[2 ; 4]$.

As we stressed in the introduction, one of the problems of modern analysis is to generalize theorems of this type to the case of differential equations in three variables. In the present paper, we investigate the conditions for the subsequence $\left\{A_{m n 0}\right\}$ of the series development $\sum_{n=0}^{\infty} \sum_{m=0}^{\infty} \sum_{r=0}^{\infty} A_{n m r} X^{n} Z^{m} Z^{* r}$ of a solution of $(2.2)$ of the class $\left({ }^{15}\right) \mathfrak{K}_{1}$ and for $s=1$, in order that the manifolds $Z=Z_{\nu}(X)$ where $\psi$ becomes infinite of the first order in a certain simple way which depends on $X$.

If we assume that

$$
\sum_{\nu=0}^{M-1} \frac{\alpha_{\nu}(X)}{Z-Z_{\nu}(X)}=\frac{1}{\sum_{\nu=0}^{M} a_{\nu} Z^{\nu}-X}, \quad a_{M} \neq 0
$$

where $\left({ }^{16}\right) a_{\nu}$ are constants, and if we denote by $h_{\nu}(X)$ the elementary symmetric functions of the $Z_{\nu}(X)$, i.e., if

(2) $h_{M-1}(X)=-\sum_{\nu=0}^{M-1} Z_{\nu}(X), \quad h_{M-2}(X)=\frac{1}{2} \sum_{\nu=0}^{M-1} \sum_{\mu=0}^{M-1} Z_{\nu}(X) Z_{\mu}(X), \cdots, \nu \neq \mu$ then

$$
h_{\nu}(X)=a_{\nu} / a_{M}, \text { for } \nu=M-1, \cdots, 1, h_{0}(X)=\frac{a_{0}-X}{a_{M}} .
$$

Multiplying (1) by $\sum_{\nu=0}^{M} h_{\nu} Z^{\nu}=\left(-X+\sum_{\nu=0}^{M} a_{\nu} Z^{\nu}\right) / a_{M}$, developing $\left(Z-Z_{\mu}(X)\right)^{-1}$ into the power series $-\sum_{\nu=0}^{\infty} Z_{\mu}^{-1-\nu} Z^{\nu}$ and comparing the coefficients on both sides of the new expression, one obtains the relation:

$$
\begin{gathered}
\sum_{\mu=0}^{M-1} \alpha_{\mu}(X) \sum_{k=0}^{\nu}\left(Z_{\mu}(X)\right)^{-(k+1)} h_{\nu-k}(X)=0, \quad \text { for } \nu=1, \cdots, M-1, \\
a_{M} \sum_{\mu=0}^{M-1} \alpha_{\mu}(X)\left(Z_{\mu}(X)\right)^{-1} h_{0}(X)=-1 .
\end{gathered}
$$

In this case we have

(2b) $\frac{\partial h_{\nu}(X)}{\partial X}=0$ for $\nu=M-1, M-2, \cdots, 1, \quad \frac{\partial h_{0}(X)}{\partial X}=-\frac{1}{a_{M}}$.

(15) For the definition of the class $\mathcal{K}_{1}$ see p. 264.

(16) We note that in the general case the $a$, depend also on $X$. 
TheOREM 4.1. Let

$$
\sum_{n=0}^{\infty} \sum_{m=0}^{\infty} \sum_{r=0}^{\infty} A_{n m r} X^{n} Z^{m} Z^{* r}
$$

be a series development around $X=Z=Z^{*}=0$ of a solution $\psi\left(X, Z, Z^{*}\right) \in \mathfrak{K}_{1}$ of the differential equation (3.1) (p. 272) where (as mentioned before) $F\left(Z, Z^{*}\right)$ is an entire function.

A. Suppose that for $\Im=\left[-\infty<X<\infty,|Z|<\infty,\left|Z^{*}\right|<\infty\right], \psi\left(X, Z, Z^{*}\right)$ has $M$ singularities of the type discussed previously. More exactly,

$$
\begin{gathered}
\psi\left(X, Z, Z^{*}\right)=\mathrm{P}_{1}\left[\sum_{\nu=0}^{M-1} \frac{\alpha_{\nu}(X)}{Z-Z_{\nu}(X)}\right]+\phi\left(X, Z, Z^{*}\right), \\
0<\left|Z_{0}(X)\right|<\left|Z_{1}(X)\right|<\cdots<\left|Z_{M-1}(X)\right|<\infty
\end{gathered}
$$

where $\phi\left(X, Z, Z^{*}\right)$ is regular in $\mathrm{J}$, the $h_{\nu}(X)$ corresponding to the $Z_{\nu}(X)$ (see (2)) satisfy the relation (2a), and the $\alpha_{\nu}(X)$ are connected with the $h_{\nu}(X)$ by the relations (3), (3a). Then for every integer $s \geqq 1$,

$$
\begin{aligned}
l_{M+s}(X) & =0, \\
l_{\mathcal{K}}(X) & \equiv \lim _{m \rightarrow \infty}\left(\left|D_{m, K^{K}}(X)\right|^{1 / m}\right) .
\end{aligned}
$$

Here

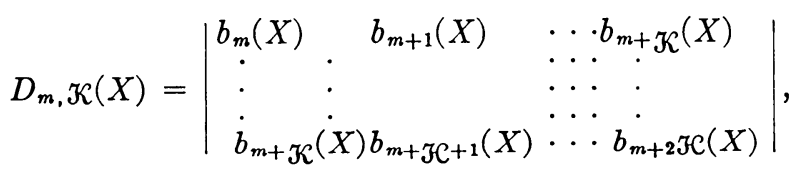

$$
\begin{aligned}
& b_{\mu}(X)=\sum_{n=0}^{\infty} A_{n \mu 0} X^{n} .
\end{aligned}
$$

Further, in this case the relations

$$
0<\lim _{m \rightarrow \infty}\left|\frac{D_{m, 0}(X)}{D_{m+1,0}(X)}\right|
$$

$$
<\cdots \lim _{m \rightarrow \infty}\left|\frac{D_{m, M-1}(X) D_{m+1, M-2}(X)}{D_{m+1, M-1}(X) D_{m, M-2}(X)}\right| \leqq \rho(X)<\infty,
$$

and

$$
\begin{aligned}
Z_{0}(X) & =\frac{1}{2} \lim \left|\gamma_{m}^{(1)}(X)\right|^{-1 / m}\left[T(X)+i\left(1-T^{2}(X)\right)^{1 / 2}\right], \\
\gamma_{m}^{(1)}(X) & =b_{m}(X) \equiv \sum_{n=0}^{\infty} A_{n m 0} X^{n}, \quad \lim \equiv \lim _{m \rightarrow \infty},
\end{aligned}
$$




$$
T(X)=-\frac{1}{t}\left[\frac{\lim \left|\sum A_{n m 0} X^{n}\right|^{1 / m}}{\left(\lim \left|\sum_{s \rightarrow m}^{\infty}\left(\begin{array}{c}
s \\
m
\end{array}\right) \sum A_{n s 0} X^{n}(-t)^{s-m}\right|^{1 / m}\right)^{2}}\right.
$$

(9b)

$$
\begin{aligned}
& \left.-\frac{1}{\lim \left|\sum A_{n m 0} X^{n}\right|^{1 / m}}\right]+t \lim \left|\sum A_{n m 0} X^{n}\right|^{1 / m}, \\
\sum & \equiv \sum_{n=0}^{\infty}, \\
\alpha_{0}(X) & =\lim _{Z \rightarrow Z_{0}(X)}\left(Z_{0}(X)-Z\right) \psi\left(X, Z, Z^{*}\right)
\end{aligned}
$$

hold. Further, if we determine successively the power series

$$
\sum_{m=0}^{\infty} \gamma_{m}^{(k)}(X) Z^{m}=\sum_{m=0}^{\infty}\left[\gamma_{m}^{(1)}(X)-\sum_{\mu=0}^{k-1} \frac{\alpha_{\mu}(X)}{\left(Z_{\mu}(X)\right)^{m+1}}\right] Z^{m}, k=2,3, \cdots M,
$$

then the expressions formed analogously to (9) and (10) with $\gamma_{m}^{(1)}(X)=\sum A_{n m 0} X^{n}$ replaced by $\gamma_{m}^{(k)}(X)$ yield $Z_{k}(X)$ and $\alpha_{k}(X), k=2, \cdots, M$.

B. Vice versa, the conditions (6), (8), (3), (3a) [with $Z_{\nu}(X)$ and $\alpha_{\nu}(X)$ determined in a way described above] are sufficient in order that for $-\infty<X^{(0)}$ $\leqq X \leqq X^{(1)}<\infty$ the solution $\psi \in \mathfrak{K}_{1}$ has the form indicated on the right hand side of (5).

Proof. A. According to our definition in $\S 2$, the associate function $g(X, Z)$ of the solution $\psi \in \varkappa_{1}$ of $(2.2)$ is given by the relation

$$
g(X, Z)=\psi(X, Z, 0),
$$

and according to the Theorem 3.1 the singularities of $\psi\left(X, Z, Z^{*}\right)$ and $g(X, Z)$ $\equiv \psi(X, Z, 0)$ coincide.

For every fixed $X, g(X, Z)$ is a function of a complex variable $Z$. According to the classical results (see $[9 ; 10 ; 17]$ ) the necessary conditions that for every $-\infty<X^{(0)} \leqq X \leqq X^{(1)}<\infty, \psi(X, Z, 0)$ has the representation

$$
\sum_{\nu=0}^{M-1} \frac{\alpha_{\nu}(X)}{Z-Z_{\nu}(X)}+\phi_{1}(X, Z)
$$

where $\phi_{1}(X, Z)$ for $X^{(0)} \leqq X \leqq X^{(1)}$ is an entire function of $Z$, is that the $l_{M+\varepsilon}(X)$ vanish for $s \geqq 1$. In this case, for the absolute value $\left|Z_{k-1}(X)\right|$ of the $k$ th pole, we obtain the relation

$$
\lim _{m \rightarrow \infty}\left|\frac{D_{m, k-1}(X) D_{m+1, k-2}(X)}{D_{m+1, k-1}(X) D_{m, k-2}(X)}\right| \equiv\left|Z_{k-1}(X)\right| .
$$


We proceed now to the determination of the argument $\theta_{0}(X)$ of the pole $Z_{0}(X)$ nearest to the origin. If we write $\zeta=Z-t, t$ real, then from the relations

$$
\left|Z_{0}\right| \sin \theta_{0}=\left|\zeta_{0}\right| \sin \beta, \quad-t+\left|Z_{0}\right| \cos \theta_{0}=\left|\zeta_{0}\right| \cos \beta
$$

we obtain

$$
\begin{aligned}
& \cos \theta_{0}=-\frac{1}{2}\left\{\frac{1}{t}\left[\frac{\left|\zeta_{0}\right|^{2}}{\left|Z_{0}\right|}-\left|Z_{0}\right|\right]-\frac{t}{\left|Z_{0}\right|}\right\} \\
& \sin \theta_{0}=\left\{1-\frac{1}{4}\left[\frac{1}{t}\left(\frac{\left|\zeta_{0}\right|^{2}}{\left|Z_{0}\right|}-\left|Z_{0}\right|\right)-\frac{t}{\left|Z_{0}\right|}\right]^{2}\right\}^{1 / 2} .
\end{aligned}
$$

Since $\psi(X, Z, 0)$ has a pole of the first order at $Z_{0}(X)$ it follows that

$$
\left|Z_{0}(X)\right| \equiv\left\{\lim \left|\sum_{n=0}^{\infty} A_{n m 0} X^{n}\right|^{1 / m}\right\}^{-1} \text {. }
$$

If we then subtract from $\sum_{n=0}^{\infty} \sum_{m=0}^{\infty} A_{n m 0} X^{n} Z^{m}$ the function $\alpha_{0}(X) /\left(Z-Z_{0}(X)\right)$ then we obtain the expression

$$
\sum_{m=0}^{\infty} \gamma_{m}^{(2)}(X) Z^{m}
$$

and using the coefficients of the series development of $\gamma_{m}^{(2)}(X)$ we determine in the same way the $\theta_{1}(X)$ and $\alpha_{1}(X)$. In the same way, using the expressions (11) we can successively determine all $Z_{\nu}(X)$ and $\alpha_{\nu}(X), \nu=2,3,4, \cdots$, $M-1$. If we substitute the values which we obtain from (2) and (2a) for $h_{M-\mathfrak{H C}}(X)$ into (3) and (3a) the relations (3) and (3a) follow.

Using a similar method, one can consider the more general case of singularities, namely, when the coefficients $a_{\nu}$ in (3.6a), p. 373 are not constants but functions of $X$. The results of the present section are valid also in this case. (Of course, now instead of (3), (3a), we have more complicated relations.) On the other hand, the proof of the convergence of the series which one obtains for $\mathrm{P}_{1}\left[\left(\sum_{\nu=0}^{M} a_{\nu}(X) Z^{\nu}\right)^{-1}\right]$ for the values of $Z$ for which $\left|\sum_{\nu=0}^{M} a_{\nu}(X) Z^{\nu}\right| \geqq \eta>0$ (see Theorem 3.1) is more difficult. (The same holds for some other theorems of §3.) We obtain for the $n$th derivative of

$$
\begin{aligned}
& L^{-1} \equiv\left(\sum_{\nu=0}^{M} a_{\nu}(X) Z^{\nu}\right)^{-1} \text { the expression } \\
&\left(L^{-1}\right)^{(n)}=(-1)^{n}\left[n ! L^{(1)^{n}} L^{-n-1}-\left\{\begin{array}{c}
n \\
n-2,2
\end{array}\right\} L^{(1)^{n-2} L^{(2)} L^{-n}}\right. \\
&+\left\{\begin{array}{c}
n \\
n-3,3
\end{array}\right\} L^{(1)^{n-3} L^{(3)} L^{-n+1}} \\
&\left.+\left\{\begin{array}{c}
n \\
n-3,2,1
\end{array}\right\}^{L^{(1)^{n-3}} L^{(2)} L^{(1)} L^{-n+1}+\cdots}\right] \\
& n=1,2, \cdots
\end{aligned}
$$


$L^{(k)} \equiv \sum_{\nu=0}^{M}\left(d^{k} a_{\nu} / d X^{k}\right) Z^{\nu}$, and

$$
\left\{\begin{array}{c}
n \\
n-\alpha, \beta, \gamma, \cdots
\end{array}\right\}
$$

are certain constants which we do not determine here. The main difficulty which arises now consists of determining upper bounds for the right hand sides of (18) and showing that the series analogous to (3.9) converges. We note that in a number of special cases this proof can be easily carried out in the same way as in the present paper.

E.g., if we assume that the $a_{\nu}(X)$ are linear functions of $X$, all terms except $(-1)^{n} n ! L^{(1)^{n}} L^{-n-1}$ on the right hand side of (18) vanish. If in this case (for the sake of simplicity) we assume that $\sigma=1$, in (3.6a), we obtain for $g^{(k)}(X, Z)$, see $(3.7)$, the expression $(-1)^{k} k ! L^{(1)^{k}} L^{-k-1}$. For points $\left(X, Z, Z^{*}\right)$, for which $|L| \geqq \eta>0$ and $\left|L^{(1)}\right| \leqq \alpha,\left|Z^{*}\right| \leqq c<\infty$, we have $\left|g^{(k)}\right| \leqq k ! \alpha^{-1} \epsilon^{-k-1}$, where $\tau=(\eta / \alpha)$ and we can repeat all considerations used in the proof of Theorem 3.1 .

\section{REFERENCES}

1. Stefan Bergman, Zur Theorie der algebraischen Potentialfunktionen des dreidimensionalen Raumes, Math. Ann. vol. 99 (1928) pp. 629-659 and vol. 101 (1929) pp. 534-558.

2. - Zur Theorie der Funktionen, die eine lineare partielle Differentialgleichung befriedigen, Rec. Math. (Mat. Sbornik) N.S. vol. 2 (1937) pp. 1169-1198.

3. - Linear operators in the theory of partial differential equations, Trans. Amer. Math. Soc. vol. 53 (1943) pp. 130-155.

4. - Certain classes of analytic functions of two real variables and their properties, Trans. Amer. Math. Soc. vol. 57 (1945) pp. 299-331.

5. - A class of harmonic functions in three variables and their properties, Trans. Amer. Math. Soc. vol. 59 (1946) pp. 216-247.

6. - On solutions with algebraic character of linear partial differential equations, Trans. Amer. Math. Soc. vol. 68 (1950) pp. 461-507.

7. Stefan Bergman and M. Schiffer, Kernel functions in the theory of differential equations, Duke Math. J. vol. 15 (1948) pp. 535-566.

8. - Kernel functions and elliptic differential equations in mathematical physics, New York, Academic Press, 1953.

9. Jacques Hadamard, Essai sur l'étude des fonctions données par leur dévelopment de Taylor, Journal de Mathématique 4th series vol. 8 (1892) pp. 101-186.

10. — La série de Taylor et son prolongement analytique, Scienta, 1901.

11. E. Kreyszig, On a class of partial differential equations, Journal of Rational Mechanics and Analysis vol. 4 (1955) pp. 907-923.

12. - On certain partial differential equations and their singularities, Journal of Rational Mechanics and Analysis vol. 5 (1956) pp. 805-820.

13. - Relations between properties of solutions of partial differential equations and the coefficients of their power series development, to appear in the Journal of Mathematics and Mechanics vol. 6 (1957).

14. M. Eichler, On the differential equation $U_{y y}+U_{x x}+N(x) U=0$, Trans. Amer. Math. Soc. vol. 65 (1949) pp. 259-278.

15. W. Magnus and F. Oberhettinger, Formeln und Sätze für die speziellen Funktionen der mathematischen Physik, Die Grundlehren der mathematischen Wissenschaften in Einzeldarstellungen vol. 52 (1948). 
16. Josephine Mitchell, Some properties of solutions of partial differential equations given by their development, Duke Math. J. vol. 13 (1946) pp. 87-104.

17. H. von Schaper, Über die Theorie der Hadamardschen Funktionen und ihre Anwendung auf das Problem der Primzahlen, Dissertation, Göttingen, 1898.

Stanford UNIVERSITY,

Stanford, Calif. 